\title{
Reacciones adversas a vacuna DPT del Programa Nacional de Inmunizaciones
}

\author{
Katia Abarca $\mathbf{V}^{\mathbf{1}}$, Jaime Cerda $\mathrm{L}^{\mathbf{2}}$, Catterina Ferreccio $\mathbf{R}^{2}$. \\ Adverse reactions to diphtheria- \\ pertussis-tetanus vaccine in the \\ Chilean Immunization Program
}

Background: During 2005, the surveillance system of the Chilean Immunization Program detected an increased number of adverse reaction notifications associated to diphtheria, pertussis and tetanus whole-cell vaccine (DPT), coincidently with a change in the vaccine manufacturer. Aim: To compare the reactogenicity of two DPT formulations (vaccines 1 and 2) in 18-month-old infants and 4-year-old children. Material and methods: Severe adverse reactions to DPT were studied at the emergency room of two hospitals of Santiago in a case-control study (110 cases and 171 controls, who consulted for other causes). Simultaneously, the incidence of total adverse reactions (mild and severe) for vaccine 1 and 2 was estimated in a cohort of 1,017 children vaccinated in an ambulatory health center of the same area. The formulation of DPT received by all participants was verified, as well as the temporal relation with consultation or symptoms referred by their caregivers. Results: There was a greater probability of consulting at the emergency rooms for severe adverse reactions among children who received vaccine 1 (odds ratio (OR) $=7.1 ; \mathrm{p}<0.001$ ), being greater among 4-year-old children ( $O R=18.9 ; p<0.001)$. Coincidently, in the cohort of vaccinated children, those who received vaccine 1 had a higher risk of presenting adverse reactions $(R R=2.9 ; p<0.001$ ), being high fever the commonest. Conclusions: We confirmed that vaccine 1 was associated to a higher risk of adverse reactions, especially among 4-year-old children. These results allowed the sanitary authority to adopt an informed decision. The usefulness of observational studies in vaccine adverse reactions is confirmed (Rev Méd Chile 2009; 137: 10-7).

(Key words: Adverse drug reaction reporting system; Bacterial vaccines; Diphtheria-tetanuspertussis vaccine)

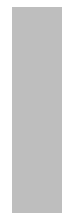

\begin{abstract}
Recibido el 8 de enero, 2008. Aceptado el 22 de septiembre, 2008.
El presente estudio contó con el financiamiento del Ministerio de Salud. La metodología, recolección de datos, análisis e interpretación de resultados y preparación del manuscrito fueron realizados exclusivamente por los autores, sin la participación del ente financiador. Departamentos de Pediatría ${ }^{1}$ y de Salud Pública ${ }^{2}$, Facultad de Medicina, Pontificia Universidad Católica de Chile. Santiago de Chile.
\end{abstract}

Correspondencia a: Catterina Ferreccio R. Departamento de Salud Pública, Pontificia Universidad Católica de Chile. Marcoleta 434, Santiago, Chile. Tel.: (56-2) 354 3038. Fax: (56-2) 6331840.

E mail: cferrec@med.puc.cl 
$E^{1}$ Programa Nacional de Inmunizaciones (PNI) del Ministerio de Salud de Chile (MINSAL) suministra los refuerzos de vacuna difteria-tétanos-pertussis de células enteras (DPT) a lactantes de 18 meses y preescolares de 4 años ${ }^{1}$. Durante el segundo semestre de 2005, el sistema de vigilancia del PNI detectó un aumento en el número de notificaciones de reacciones adversas asociadas a la vacunación DPT, preferentemente en preescolares de 4 años, coincidente con un cambio en el laboratorio manufacturador de la vacuna. Con el objetivo de caracterizar y estimar la magnitud de esta situación, el MINSAL solicitó a una institución universitaria un estudio de campo, cuya información sirviera de sustento técnico para la toma de decisiones. Durante el año 2005 se usaron como refuerzos DPT para los niños de 18 meses y 4 años dos formulaciones de vacuna, permitiendo realizar una comparación directa entre ambos productos y determinar si efectivamente hubo un aumento significativo de reacciones adversas. Para ello, se realizaron paralelamente dos estudios epidemiológicos observacionales, cuya metodología y resultados se presentan a continuación. Con el fin de proteger la identidad de los laboratorios manufacturadores, se denominó vacuna 1 y vacuna 2 a las formulaciones usadas como refuerzo DPT durante el año 2005.

\section{PACIENTES Y MÉTODOS}

Se diseñó un estudio de casos y controles de reacciones adversas a vacuna en niños consultantes entre enero y diciembre de 2005 en dos servicios de urgencia y un estudio de cohorte retrospectiva de incidencia de reacciones adversas en niños que recibieron la vacuna DPT durante 2005 (Figura 1). Ambos estudios se realizaron en establecimientos pertenecientes al Servicio de Salud Metropolitano Suroriente (SSMSO) y contaron con la aprobación de los comités de ética de la Facultad de Medicina de la Pontificia Universidad Católica de Chile y del MINSAL.

\begin{tabular}{|c|c|}
\hline Estudio de casos y controles & Estudio de cohorte \\
\hline $\begin{array}{l}\text { Permiten estudiar efectos esperados, } \\
\text { especialmente aquellos severos } \\
\text { y de baja frecuencia }\end{array}$ & $\begin{array}{l}\text { Permiten estudiar todos los efectos, } \\
\text { conocidos y desconocidos, en particular } \\
\text { aquellos más frecuentes }\end{array}$ \\
\hline $\begin{array}{l}\text { Requiere de pocos sujetos para encontrar } \\
\text { un riesgo significativo atribuible a la exposición }\end{array}$ & $\begin{array}{l}\text { Necesita un gran número de sujetos a } \\
\text { visitar para encontrar algunos casos }\end{array}$ \\
\hline $\begin{array}{l}\text { Muestra: casos de la enfermedad y controles } \\
\text { sin la enfermedad o problema de salud }\end{array}$ & $\begin{array}{l}\text { Muestra: un grupo completo de la población, } \\
\text { definido por alguna característica en común (cohorte). }\end{array}$ \\
\hline $\begin{array}{l}\text { Rol en este estudio: identificar problemas graves } \\
\text { e infrecuentes }\end{array}$ & $\begin{array}{l}\text { Rol en este estudio: identificar todos los problemas } \\
\text { asociados a las vacunas que ocurren con frecuencias } \\
\text { mayores a } 1 \text { por } 1.000 \text { casos vacunados }\end{array}$ \\
\hline $\begin{array}{l}\text { Lugar y tiempo: Servicios de Urgencia, } 2005 . \\
\text { Casos: Consultantes por reacciones adversas a vacuna } \\
\text { Grupo de comparación: Consultantes por otros } \\
\text { problemas de salud, de igual edad }\end{array}$ & $\begin{array}{l}\text { Lugar y tiempo: Area de cobertura del consultorio, } 2005 \\
\text { Casos: Niños en que se constató alguna reacción } \\
\text { post-vacuna } 1 \text { ó } 2 \\
\text { Grupo de comparación: Diferente formulación de vacuna } \\
\text { (1 vs 2) }\end{array}$ \\
\hline Exposición: tipo y lote de vacuna DPT recibida & Exposición: tipo y lote de vacuna DPT recibida \\
\hline Medida de riesgo calculada: Odds ratio & Medida de riesgo calculada: Riesgo relativo \\
\hline
\end{tabular}

Figura 1. Características principales de los dos diseños de investigación observacionales utilizados en el presente estudio. 
Estudio de casos y controles. Se realizó en los servicios de urgencia infantil de los hospitales Sótero del Río y Padre Hurtado (SSMSO) en base al análisis de las consultas efectuadas entre enero y diciembre de 2005. Tanto los casos potenciales como los controles fueron identificados a partir de los registros electrónicos de las consultas de urgencia.

- Definición de caso: Niños entre 18-20 meses y 4-5 años de edad que consultaron en los 7 días post-vacunación presentando uno o más de los siguientes signos clínicos: convulsiones, encefalopatía, episodio de hipotonía-hiporrespuesta, celulitis o inflamación del brazo, convulsión febril, llanto persistente y anafilaxia. También se incluyeron aquellos consultantes en quienes el médico no especificó los signos clínicos, pero registró el diagnóstico de "reacción post-vacuna". Los signos clínicos detallados han sido descritos en la literatura como reacción adversa a vacuna $\mathrm{DPT}^{2-6}$.

- Definición y selección de controles: Por cada caso se seleccionó 1-2 controles pareados por edad entre aquellos niños que consultaron en el mismo día y centro asistencial por cuadros clínicos claramente no asociables a reacciones adversas a vacunación DPT (diagnósticos traumatológicos, quirúrgicos o médicos específicos no sindromáticos). Se excluyeron niños con cuadros febriles o dermatológicos inespecíficos.

- Asignación de la vacuna: En forma ciega (i.e. sin conocer su calidad de caso o control) se consignó la formulación de vacuna recibida por cada niño a partir de los datos disponibles en sus consultorios de origen, registrándose la fecha de vacunación y el lote de la vacuna. Sólo se incluyeron en el análisis los niños que contaban con información de su vacuna, cuya proporción fue similar en casos y controles (50,2\% y $55,2 \%$, respectivamente).

Se calculó el tiempo transcurrido entre la fecha de vacunación y la fecha de consulta, siendo considerado como período atribuible a la vacunación una ventana de tiempo vacunación-consulta inferior a siete días. Finalmente, se identificó cada lote según el laboratorio manufacturador de la vacuna y se cruzó la condición de caso y control con el lote de vacuna recibida, calculando el odds ratio (OR) según vacuna. El análisis se realizó para el total de niños y diferenciado por grupos de edad.

\section{Estudio de cohorte retrospectiva}

- Definición de la cohorte: Se identificó la cohorte de todos los niños que recibieron la vacuna DPT a los 18 meses y 4 años durante el año 2005 en el vacunatorio del Centro de Salud Familiar Bernardo Leighton (SSMSO).

- Asignación de la vacuna: De los registros de vacunación del consultorio se obtuvo para cada niño la fecha de nacimiento, domicilio, teléfono, fecha de vacunación y lote de vacuna recibida.

- Detección de reacciones adversas en la cohorte: Los datos de los integrantes de la cohorte fueron analizados retrospectivamente para determinar si habían presentado una reacción adversa a vacuna con posterioridad a la aplicación de la vacuna DPT. Al igual que en el estudio de caso-control, se consideró reacción post-vacuna a la ocurrencia post-vacunación de fiebre alta, convulsiones, encefalopatía, episodio de hipotonía-hiporrespuesta, celulitis o inflamación del brazo, convulsión febril, llanto persistente (más de 3 horas) y anafilaxia, registrándose además si estos síntomas motivaron una consulta médica u hospitalización ${ }^{2-6}$. La información referente a "fiebre alta" fue recolectada en términos cualitativos, dada la naturaleza del diseño de investigación, basado en el recuerdo de las madres o cuidadores.

Enfermeras capacitadas contactaron telefónicamente o mediante visita domiciliaria a las madres o cuidadores de cada niño, aplicándoles una encuesta estandarizada sobre la ocurrencia de síntomas y signos relacionados a la vacunación DPT. Se interrogó sobre el tiempo transcurrido entre la vacunación y el inicio de los síntomas y signos, su duración y si motivaron consulta médica, junto a su correspondiente diagnóstico. Se determinó la tasa de incidencia y el riesgo relativo (RR) de presentar reacciones adversas a vacunación DPT según la edad del niño y la formulación de vacuna recibida. El estudio fue conducido en forma ciega tanto por quienes realizaron las entrevistas como por quienes participaron en el análisis de los datos. Sólo una vez completado el análisis se asignó la identidad del laboratorio de origen de cada lote de vacuna. 


\section{RESUlTADOs}

Estudio de casos y controles. Se identificaron 219 niños que consultaron por un cuadro compatible con reacción adversa post-vacuna y 310 controles. Se pudo comprobar el antecedente de vacunación (vacuna y lote) en 110 casos (50,2\%) y en 171 controles (55,2\%). El 25,3\% de los niños estudiados recibió la vacuna 1 , el resto recibió la vacuna 2 . Los casos recibieron la vacuna 1 con mayor frecuencia que los controles (33,6\% y 19,9\%, respectivamente), siendo el riesgo de presentar un cuadro compatible con reacción adversa a DPT dos veces mayor entre los niños que recibieron la vacuna 1 en comparación con los que recibieron la vacuna 2 ( $\mathrm{OR}=2,0$; IC 95\% 1,1-3,7; $\mathrm{p}<0,01$ ) (Tabla 1). El OR de la vacuna 1 respecto de la vacuna 2 aumenta a 7,1 al considerar sólo las reacciones adversas temporalmente asociadas a la vacunación DPT (i.e.: cuadro clínico compatible más vacunación en los 7 días previos) ( $p<0,001$ ). Según edad, el OR fue 3,1 a los 18 meses $(p=0,08)$ y 18,9 a los 4 años ( $p$ $<0,001$ ) (Tabla 1). El riesgo atribuible a la vacuna 1 fue $86 \%$ en el total de niños y $95 \%$ en los niños de 4 años. La mayoría de los motivos de consulta 0 diagnósticos de quienes recibieron la vacuna 1 fueron problemas locales, siendo menos frecuentes los síntomas generales ( $64 \%$ y $36 \%$, respectivamente). Entre los niños que recibieron la vacuna 2 hubo menos reacciones adversas, presentado igual frecuencia las reacciones generales (50\%) y locales (50\%) (Tabla 2).

Estudio de cohorte retrospectiva. Durante 2005 se vacunaron 1.260 niños con refuerzo de DPT en el consultorio seleccionado, logrando entrevistarse a 1.017 (80,7\%), 478 niños de 18 meses y 539 niños de 4 años. En 702 casos (69,0\%) la entrevista fue contestada por la madre del niño; $441(43,4 \%)$ entrevistas fueron realizadas telefónicamente y $576(56,6 \%)$ mediante visitas domiciliarias.

Un total de 141 niños (13,9\%) presentaron reacciones adversas post-vacunación, 89 (16,5\%) correspondientes a niños de 4 años y $52(10,9 \%)$ a niños de 18 meses, siendo el $R R=1,5$ para los niños de 4 años respecto a los niños de 18 meses (IC 95\% 1,1-2,1; $\mathrm{p}<0,01$ ). La Tabla 3 resume los tipos de reacciones adversas más frecuentemente encontrados según grupo de edad, siendo la más

Tabla 1. Vacuna recibida por consultantes de 18 meses y 4 años por reacciones adversas a vacuna. Servicios de U rgencia SSM SO (2005)

\begin{tabular}{|c|c|c|c|c|c|}
\hline \multicolumn{6}{|c|}{ Niños consultantes entre enero y diciembre 2005} \\
\hline & Casos & Controles & OR & IC $95 \%$ & valor $p$ \\
\hline Vacuna 1 & 37 & 34 & 2,0 & $1,1-3,7$ & $<0,01$ \\
\hline Vacuna 2 & 73 & 137 & 0,5 & $0,3-0,9$ & $<0,01$ \\
\hline \multicolumn{6}{|c|}{ Niños consultantes en los primeros 7 días post-vacunación } \\
\hline & Casos & Controles & OR & IC $95 \%$ & valor $p$ \\
\hline \multicolumn{6}{|l|}{ Total } \\
\hline Vacuna 1 & 14 & 34 & 7,1 & $2,5-20,2$ & $<0,001$ \\
\hline Vacuna 2 & 8 & 137 & 0,1 & $0,05-0,4$ & $<0,001$ \\
\hline \multicolumn{6}{|l|}{18 meses } \\
\hline Vacuna 1 & 6 & 23 & 3,1 & $0,7-13,2$ & 0,08 (ns) \\
\hline Vacuna 2 & 5 & 59 & 0,3 & $0,1-1,4$ & 0,08 (ns) \\
\hline \multicolumn{6}{|l|}{4 años } \\
\hline Vacuna 1 & 8 & 11 & 18,9 & $3,7-108,6$ & $<0,001$ \\
\hline Vacuna 2 & 3 & 78 & 0,05 & $0,01-0,3$ & $<0,001$ \\
\hline
\end{tabular}

Fuente: Hospital Padre Hurtado y Sótero del Río (SSMSO, 2005). 
Tabla 2. M otivo de consulta o diagnóstico de los casos asociados a DT P entre los consultantes de dos servicios de urgencia. SSM SO (2005)

\begin{tabular}{|llcc|}
\hline Edad & Diagnóstico & Vacuna & Días post vacunación \\
\hline 18 meses & Inflamación del brazo & 1 & 1 \\
4 años & Inflamación del brazo y dolor & 1 & 2 \\
4 años & Inflamación del brazo & 1 & 2 \\
4 años & Inflamación del brazo & 1 & 1 \\
4 años & Inflamación del brazo y vómitos & 1 & 3 \\
18 meses & Dolor del brazo y fiebre & 1 & 2 \\
4 años & Dolor del brazo, impotencia funcional y fiebre & 1 & 1 \\
18 meses & Celulitis del brazo y fiebre & 1 & 1 \\
4 años & Celulitis del brazo y codo & 1 & 2 \\
4 años & Fiebre y molestias post vacunación & 1 & 2 \\
18 meses & Fiebre y llanto persistente & 1 & 2 \\
18 meses & Fiebre y molestias post vacunación & 1 & 2 \\
18 meses & Fiebre y lipotimia & 1 & 1 \\
4 años & Reacción post vacuna & 1 & 2 \\
18 meses & Inflamación y dolor del brazo & 2 & 2 \\
4 años & Celulitis del brazo & 2 & 2 \\
4 años & Celulitis del brazo & 2 & 1 \\
4 años & Dolor del brazo & 2 & 1 \\
18 meses & Fiebre y cianosis post vacunación & 2 & 4 \\
18 meses & Convulsión febril & 2 & 7 \\
18 meses & Convulsión febril & 2 & 2 \\
18 meses & Convulsión febril & 2 & 2 \\
\hline
\end{tabular}

Fuente: Hospital Padre Hurtado y Sótero del Río (SSMSO, 2005).

Tabla 3. Frecuencia y tasa de reacciones adversas reportadas en la cohorte de niños vacunados durante 2005. Centro de Salud Familiar Bernardo Leighton (SSM SO)

\begin{tabular}{|c|c|c|c|c|}
\hline $\mathbf{R A}(*)$ & $\begin{array}{c}\text { Total } \\
n(\%) n=1.017\end{array}$ & $\begin{array}{c}18 \text { meses } \\
n(\%) n=478\end{array}$ & $\begin{array}{r}4 \\
\text { n }(\%\end{array}$ & $\begin{array}{l}\text { años } \\
n=539\end{array}$ \\
\hline Fiebre alta & $95(9,3)$ & $33(6,9)$ & 62 & $(11,5)$ \\
\hline Inflamación importante del brazo & $71(7,0)$ & $24 \quad(5,0)$ & 47 & $(8,7)$ \\
\hline Llanto persistente & $18(1,8)$ & $6(1,3)$ & 12 & $(2,2)$ \\
\hline Convulsiones & $5(0,5)$ & $0 \quad(0,0)$ & 5 & $(0,9)$ \\
\hline Hipotonía-hiporrespuesta & $0(0,0)$ & $0 \quad(0,0)$ & 0 & $(0,0)$ \\
\hline Anafilaxia & $0(0,0)$ & $0 \quad(0,0)$ & 0 & $(0,0)$ \\
\hline Celulitis & $0(0,0)$ & $0 \quad(0,0)$ & 0 & $(0,0)$ \\
\hline Total & $189(18,6)$ & $63(13,2)$ & 126 & $(23,4)$ \\
\hline
\end{tabular}

*Se reporta el número de reacciones adversas (RA), no el número de niños que las presentaron. 
común en ambos grupos etarios la fiebre elevada. Las reacciones adversas se iniciaron mayoritariamente en las primeras 24 horas post-vacunación (70\%) y su duración fue inferior a una semana en 87\% de los afectados. En 48 niños (24,4\%) los síntomas motivaron una consulta médica.

Un total de $337(33,1 \%)$ niños recibieron la vacuna 1 y $680(66,9 \%)$ la vacuna 2 . El riesgo de presentar reacciones adversas fue 2,9 veces mayor entre los niños que recibieron la vacuna 1 , en comparación a los que recibieron la vacuna 2 (p $<0,001$ ). Según grupos de edad, el $R R=2,6$ en los niños de 18 meses y $\mathrm{RR}=3,0$ en los niños de 4 años (Tabla 4). Entre las reacciones adversas destaca el riesgo de presentar llanto persistente con la vacuna 1 en niños de 18 meses (RR 11,3; IC 95\% 1,3-95,5) (Tabla 4).

\section{DisCUSIÓN}

El presente estudio confirma que la vacuna DPT de células enteras produce una elevada tasa de reacciones adversas y que éstas pueden variar en cuanto a magnitud y tipo entre productos de diferentes laboratorios manufacturadores, en concordancia a lo descrito internacionalmente ${ }^{7}$. La vacuna DPT acelular produce una menor tasa de reacciones adversas ${ }^{8-9}$, sin embargo, su elevado costo la ha mantenido fuera de los programas de inmunización en la mayoría de los países en vías de desarrollo ${ }^{10}$. El grado de "tolerancia" frente a reacciones adversas a vacunas por parte de la población y sus autoridades sanitarias puede variar entre países y en un mismo país a través del tiempo, según cambien sus opciones y expectati-

Tabla 4. Incidencia de reacciones adversas reportadas en la primera semana post-vacuna D PT en la cohorte de niños vacunados durante 2005. Centro de Salud Familiar Bernardo Leighton (SSM SO)

\begin{tabular}{|c|c|c|c|c|}
\hline Edad & Vac. 1 ( $n=337)$ & Vac. $2(n=680)$ & $\mathbf{R R}$ & IC 95\% \\
\hline Total & 83 & 58 & 2,9 & $2,1-3,9$ \\
\hline 18 meses & 28 & 24 & 2,6 & $1,6-4,4$ \\
\hline \multirow[t]{2}{*}{4 años } & 55 & 34 & 3,0 & $2,0-4,4$ \\
\hline & & 18 meses & & \\
\hline RA & Vac. 1 ( $\mathrm{n}=147$ ) & Vac. 2 ( $\mathrm{n}=331)$ & $\mathrm{RR}$ & IC 95\% \\
\hline Fiebre alta & 17 & 16 & 2,4 & $1,2-4,6$ \\
\hline Inflamación $^{\dagger}$ & 17 & 7 & 5,5 & $2,3-12,9$ \\
\hline Llanto"t & 5 & 1 & 11,3 & $1,3-95,5$ \\
\hline Convulsiones & 0 & 0 & - & - \\
\hline \multirow[t]{2}{*}{ Consulta $^{\ddagger}$} & 9 & 10 & 2,0 & $0,8-4,9$ \\
\hline & & 4 años & & \\
\hline RA & Vac. 1 (n =190) & Vac. $2(\mathrm{n}=349)$ & $\mathrm{RR}$ & IC 95\% \\
\hline Fiebre alta & 37 & 25 & 2,7 & $1,7-4,4$ \\
\hline Inflamación $^{\dagger}$ & 35 & 12 & 5,4 & $2,9-10,1$ \\
\hline Llanto \# & 8 & 4 & 3,7 & $1,1-12,0$ \\
\hline Convulsiones & 1 & 4 & 0,5 & $0,1-4,1$ \\
\hline Consulta $^{\ddagger}$ & 18 & 11 & 3,0 & $1,5-6,2$ \\
\hline
\end{tabular}

$\dagger=$ inflamación importante del brazo; ${ }^{\dagger}=$ llanto persistente; ${ }^{\ddagger}=$ consulta médica por RA.

$\mathrm{RA}=$ reacción adversa; Vac. $=$ vacuna 
vas. En este sentido, la presente investigación aportó información relevante para la toma de decisiones respecto a la continuación del uso de una determinada formulación de vacuna.

Las dos metodologías empleadas mostraron en forma independiente que una de las formulaciones de vacuna se asoció a un mayor riesgo de presentar reacciones adversas, específicamente fiebre, signos inflamatorios locales, llanto persistente y necesidad de consultas médicas. No se pudo demostrar diferencias en eventos de rara ocurrencia, tales como episodios de hipotoníahiporrespuesta, encefalopatía y anafilaxia, debido a que no se detectaron en la muestra estudiada. Las reacciones adversas ocurrieron con mayor frecuencia en niños de 4 años, hecho concordante con lo reportado por diversos estudios de vacuna DPT, los cuales muestran un aumento de la reactogenicidad con la edad ${ }^{3-5}$. Usando diferentes metodologías y sujetos de análisis, es destacable la consistencia de los hallazgos en cuanto al tipo de reacciones adversas, el grupo de edad más afectado y la significancia estadística de los resultados, los cuales ratificaron lo que el sistema de vigilancia del MINSAL había detectado. La Figura 1 resume las principales diferencias entre los dos diseños observacionales utilizados en el presente estudio.

Por no contar con registros de la temperatura de los niños ingresados al estudio, no fue posible determinar si se superó la tasa esperada de fiebre mayor a $40,5^{\circ} \mathrm{C}$ reportada por el CDC $(0,3 \%)^{11}$. De igual forma, al no contar con medición del tamaño de las lesiones, tampoco fue posible determinar si se superó la tasa esperada de eritema o edema mayor a $10 \mathrm{~cm}$ reportada por un estudio canadiense $(6 \%)^{12}$. En los registros del Vaccine Adverse Event Reporting System (VAERS) estadounidense existe una entidad clínica que se ha denominado "entire limb swelling" (edema de la extremidad completa), que corresponde a una inflamación de la extremidad de origen no infeccioso, secundaria a la administración de diversas vacunas, especialmente aquellas que contienen el componente pertussis. El cuadro se presenta con mayor frecuencia luego de numerosas dosis de vacuna $\mathrm{DPT}^{13}$. Este diagnóstico no fue registrado como tal en nuestra serie, sin embargo, podría estar incluido en los casos de "inflamación importante del brazo", el cual presentó en el estudio de cohorte una mayor incidencia en niños de 4 años en comparación a los de 18 meses $^{13}$. La tasa de llanto persistente de la vacuna 1 fue superior a la tasa esperada por el CDC (1\%) ${ }^{11}$. Ambas vacunas superaron las tasas para convulsiones reportadas por el CDC y OPS (0,06\%), mientras que reacciones adversas más severas tales como episodios de hipotonía-hiporrespuesta, anafilaxia y encefalitis (cuyas tasas reportadas internacionalmente son de 6 por 10.000 , 2 por 100.000 y 1 por 1.000 .000 , respectivamente) ${ }^{6}$ no fueron observadas en la cohorte, hecho esperable para un grupo constituido por poco más de 1.000 niños.

Los resultados obtenidos presentan limitaciones propias de estudios observacionales. En el estudio de casos y controles, una primera limitante correspondió a la fuente de los casos, la cual no representa el universo de niños que consultan por reacciones adversas a vacunación DPT, ya que un número indeterminado de ellos lo hace en otros centros de salud, tanto públicos como privados. A pesar de ello, los niños que presentan reacciones adversas más severas inevitablemente consultan en los servicios de urgencia en forma directa 0 bien son derivados allí desde otros centros. Una segunda limitante fue el alto índice de falta de información en las fichas de atención de urgencia (cercano a 50\%) debido a problemas inherentes a los sistemas con registro caligráfico. Sin embargo, al ser esta limitante aleatoria, tanto casos como controles se ven afectados, así como los niños que recibieron una u otra formulación de vacuna DPT, no constituyendo un sesgo que invalide los resultados. Por su parte, el estudio de cohorte retrospectiva presentó la limitante de haber incluido un sólo consultorio, el cual puede no ser representativo de la variabilidad de las reacciones adversas a vacunación DPT de la población chilena. De todos modos, no es esperable una gran diferencia en las respuestas a vacuna en niños sanos a menos que las reacciones adversas se deban a variaciones importantes en la aplicación de las vacunas, tales como fallas puntuales de administración o en la cadena de frío. Esto no sería concordante con la información recopilada por el sistema de vigilancia del PNI, dado que éste detectó un aumento de los reportes de reacciones adversas en distintos servicios de salud a lo largo del país. Una segunda limitante del estudio de cohorte retrospectiva radica en que los datos 
recopilados se basaron fundamentalmente en la memoria de la madre o del cuidador del niño; no obstante, es razonable pensar que en general las madres o cuidadores no olvidan hechos significativos como síntomas llamativos post-vacunación. Finalmente, el hecho de haberse administrado la vacuna 1 entre los meses de agosto y diciembre de 2005 y la vacuna 2 entre los meses de enero y julio del mismo año mejoraría el recuerdo de la vacuna 1, de más reciente aplicación, eventualidad que podría haber sobreestimado los efectos adversos relacionados a la vacuna 1 .

En conclusión, ambos estudios encontraron una significativa mayor tasa de reacciones adversas con la vacuna 1 , sustentando la alerta detectada por el sistema de vigilancia del MINSAL. La formulación de vacuna introducida más tardíamente (vacuna 1) produjo más reacciones adversas, en especial inflamaciones severas del brazo,

\section{REFERENCIAS}

1. Valenzueia MT, O'Ryan M. Logros y desafíos del Programa Ampliado de Inmunizaciones en la Región de las Américas. Rev Méd Chile 2000; 128: 911-22.

2. Blumberg DA, Lewis K, Mink CM, Christenson PD, ChATFIeld P, CHERRY JD. Severe reactions associated with diphtheria-tetanus-pertussis vaccine: detailed study of children with seizures, hypotonic-hyporesponsive episodes, high fevers, and persistent crying. Pediatrics 1993; 91: 1158-65.

3. Long SS, De Forest A, Pennridge Pediatric Associates, SMith DG, LazARo C, Wassiak SGF. Longitudinal study of adverse reactions following diphtheria-tetanus-pertussis vaccine in infancy. Pediatrics 1990; 85: 294-302.

4. Baraff LJ, Cody CL, CherRY JD. DPT-associated reactions: an analysis by injection site, manufacturer, prior reactions and dose. Pediatrics 1984; 73: 31-6.

5. Cody CL, Baraff LJ, Cherry JD, Marcy SM, Manclark $\mathrm{CR}$. The nature and rate of adverse reactions associated with DPT and DT immunization in infants and children. Pediatrics 1981; 68: 650-60.

6. Pan American Health Organization. How to address events allegedly attributable to vaccination or immunization? Disponible en: http://www.who.int [Consultado el 13 de agosto de 2007].

7. Steinhoff M, Reed G, Decker M, Edwards K, Englund J, Pichichero M ET AL. A randomized comparison of reactogenicity and immunogenicity of two wholecell pertussis vaccines. Pediatrics 1995; 96: 567-70.

8. Pichichero M, Deloria M, Rennels M, Anderson E, Edwards K, Decker M et al. A safety and immunoge- mayoritariamente en los niños de 4 años. La implementación de estudios prospectivos que evalúen en forma más rigurosa las reacciones adversas a vacunas así como el perfeccionamiento de los sistemas de vigilancia ${ }^{14}$ permitirá avanzar en el objetivo final, cual es el utilizar esta valiosa herramienta preventiva con la mayor seguridad posible.

\section{Agradecimientos}

Agradecemos a la Dra. María Inés Gómez (Subdirectora Médica del Hospital Padre Hurtado), a la Dra. Mildred Rojas (Directora del Centro de Salud Familiar Bernardo Leighton) y al personal de ambas instituciones por su colaboración en la organización y entrega de la información solicitada, así como a la Srta. Alessandra Gederlini (Magíster en Estadística) por su ayuda en el análisis estadístico.

nicity comparison of 12 acellular pertussis vaccines and one whole-cell pertussis vaccine given as a fourth dose in 15 to 20-month-old children. Pediatrics 1997; 100: 772-88.

9. Haiperin S, Scheifele D, Barreto L, Pim C, Guasparini R, MEDd L ET AL. Comparison of a fifth dose of a five component acellular or a whole cell pertussis vaccine in children four to six years of age. Pediatr Infect Dis J 1999; 18: 772-9.

10. Ekwueme DU, Strebel PM, Hadier SC, Meltzer Mi, ALEN JW, Livengood JR. Economic evaluation of use of diphtheria, tetanus, and acellular pertussis vaccine or diphtheria, tetanus, and whole-cell pertussis vaccine in the United States, 1997. Arch Pediatr Adolesc Med 2000; 154: 797-803.

11. Center for Diseases Control and Prevention (CDC). Diphtheria, Tetanus and Pertussis: Recommendations for vaccine use and other preventive measures. Recommendations of the Immunization Practices Advisory Committee (ACIP). MMWR 1991; 40 (RR10): 1-28.

12. Scheifeie DW, Mefrison W, Grace M, Barreto L, Carter AO, MTtCHEL L ET AL Adverse reactions to the preschool (fifth) dose of adsorbed diphtheria-pertussis-tetanus vaccine in Canadian children. CMAJ 1991; 145: 641-7.

13. Woo E, Burwen D, Gatumu S, Ball R and the Vaccine Adverse Event Reporting System (VAERS) Working GRouP. Extensive limb swelling after immunization: reports to the Vaccine Adverse Event Reporting System. Clin Infect Dis 2003; 37: 351-8.

14. AbarCa K. Vigilancia de eventos adversos a vacunas: adverse events surveillance. Rev Chil Infectol 2007; 24: 53-6. 